\title{
Implementation of a Wiki-based Information and Communication System for Academia Europaea
}

\author{
Ilire Hasani-Mavriqi ${ }^{1}$, Helmut Leitner ${ }^{1}$, Denis Helic ${ }^{2}$ \\ and Hermann Maurer ${ }^{1}$ \\ ${ }^{1}$ Institute for Information Systems and Computer Media (IICM), Graz University of Technology, Austria \\ ${ }^{2}$ Institute for Knowledge Management (KMI), Graz University of Technology, Austria
}

In recent years, online collaboration tools such as wikis have experienced a tremendous expansion. Their success and popularity is due to their simple and efficient content creation facilities. Moreover, the formatting syntax is basically easy to learn allowing users to easily create and edit web content and thus share knowledge with each other.

Despite these advantages, the content creation process is still obscure if authors belong to a computer inexperienced user group. In this paper we present our experiences, challenges, and problems with a specific application of a wiki-based system called the Academia Europaea Information and Communication System. Furthermore, key solutions for bypassing user interaction barriers are discussed throughout this paper.

Keywords: wikis, open collaboration, web accessibility

\section{Introduction}

In recent years, the popularity of wikis as tools for knowledge sharing and collaboration has increased [8]. Wikis have become very popular particularly for their openness and ease of use. In particular, wiki content can be added and edited by everyone. An interesting research topic emerged in the last couple of years: investigation of the degree of user participation and user motivation in such a collaborative authoring environment $[3,5,7,11,12]$. Among other topics, authoring or editing content by volunteers is one of the most interesting topics discussed by scientists in this area $[1,11]$.

For instance, a considerable amount of research is conducted to measure author contribution in
Wikipedia $[1,3,11,12]$ - the most well-known online encyclopedia. In general, such studies show results such as: which user group contributes the most $[11,12]$ or how user groups are distributed among different categories in Wikipedia [3].

A group of researchers of Palo Alto Research Center [11] analyzed overall activities in Wikipedia with the focus on editing activities such as new page creation, adding/modifying/removing contents in existing pages, and reverted editing. Firstly, it was shown that there has been a general slowdown in Wikipedia editing activities in the last two years. Editors were divided into classes based on the number of their contributions per month and editing activities within each class were investigated. In Wikipedia, a small group of "elite" editors contributes a large amount of edits while the larger group of "normal" users contributes the rest. It has turned out that editing activities of the editor's class with the most edits per month did not decrease in the last two years, whereas the editors belonging to other classes decreased their overall editing activities.

Mechanisms have been implemented in order to calculate the degree of co-authorship for a given pair of authors in Wikipedia [12]. These methods are useful to discover experts belonging to a certain field. Further research [3] shows that experts in certain fields contribute to topics related to that field, but also interact strongly with other categories. 
However, to the best of our knowledge, there is a lack of research discussing the difficulties faced by a particular group of computer inexperienced contributors trying to add, edit, and publish content in a wiki system.

The aim of this paper is to fill this gap and to address these issues by discussing problems we experienced, together with the solutions we introduce, during the development of a wiki-based system - the Academia Europaea (AE for short) known as AE-Info [2]. Despite the fact that AE-Info is still on its early days, user participation is very low. Main problems identified so far range from difficulties that users of AE-Info experience during the authentication process to obstacles that they face during content creation process.

The remainder of this paper is organized as follows: Section 2 presents shortly the context of AE-Info and its users. Section 3 gives an overview and a definition of wikis. Section 4 introduces the AE-Info platform. Implementation details and solutions to the problems presented in previous sections are described in Section 5. An overview of the current status of the AE-Info is provided in Section 6. The paper is concluded by discussing steps for the future work in the same section.

\section{AE-Info Context}

The target audiences of AE-Info are members of Academia Europaea. The Academia members are scientists and leading experts in different disciplines. Most of them have a long academic career; therefore, average age is high. Moreover, many have little experience with computers and computer skills.

In general, two problems have been identified at the beginning of our endeavor: user motivation (e.g. how to motivate users to participate in the system) and usability/accessibility issues. Since our target audience experiences technical difficulties while performing basic actions such as user authentication or editing wiki pages, user contribution in AE-Info cannot be directly compared to other existing wiki systems.

For instance, the contribution measurement methods discussed in e.g. [3] or [12] could not be applied in our system without considering usability problems which our target user group faces. How difficult is it for this user group to discuss and collaborate online? How can this target audience be motivated and encouraged to participate and contribute in a collaborative authoring environment? The remainder of this paper analyses these issues in more detail and provides possible solutions to them.

A group of researchers [5] conducted a study in a corporate context which proved that employees are stimulated to contribute much more in a social-network site when their work is rewarded by points. We believe that due to AE-Info context, this concept is not applicable to our system.

Motivation seems not to be a problem among students who use wiki tools for learning. Studies $[7,4]$ show that students are very motivated to contribute in wiki-based learning environments. However, they are challenged by large number of wiki articles and authors, or they do not feel comfortable editing the content that their peers have created [7].

In general, there are studies which examine the degree of web accessibility by older people [10], but there is a lack of usability studies in the context of wikis and elderly people in particular.

As it can be noted from the overview of studies given above, many aspects and factors that may impact collaborative authoring environments are considered, but one of the most relevant factors is left aside: do users possess the knowledge of creating or editing a wiki page.

Thus, this paper will contribute to the community by showing how to implement a more accessible, useful, and engaging wiki system and by providing first accessibility results.

\section{What is a Wiki?}

Before discussing our wiki-based system in more details, a brief overview of wikis in general is given below.

The following citation is considered as a standard definition of a wiki:

"Wikis offer tremendous potential to capture knowledge from large groups of people, making tacit, hidden content explicit and widely available. They also efficiently connect those with information to those seeking it”. [8] 
The initial goal of the wiki technology was to enable users to quickly publish content on the web. Typical user activities which characterize a wiki system are: add and edit content, view content, organize and structure content, link it with other related content, access a history of contributed content [8].

The openness of wikis and their concept of "wisdom of the crowd" drive them into knowledge sharing tools. The contribution of many users with different backgrounds and specialties provides means for absorbing the knowledge from those who have it and present it to those who need it [8].

Successful applications of wikis can be found in different areas. Enterprise wikis present one of the wiki applications, which can be publicly accessible or closed only for organizational usage. Wikis in higher education present another context in which wikis can be used. Online encyclopedias are most well-known and largest wiki systems, with Wikipedia as the most famous one.

\section{AE-Info System}

The AE-Info System presents an information and communication platform for Academia Europaea which was founded in 1988 as a European, non-governmental association acting as an Academy. The members of Academia Europaea are scientists and scholars who collectively aim to promote learning, education and research. Currently with over 2400 members, Academia Europeaea brings together individual leading experts from the physical sciences and technology, biological sciences and medicine, mathematics, the letters and humanities, social and cognitive sciences, economics and the law. [2]

The purpose of AE-Info System is two folded: first, to present the achievements and activities of Academia Europaea and its members to other members, various GOs and NGOs, and the general public and second, to enhance communication between members and between members and general public.

The wiki philosophy provides mechanisms to engage members to add and edit content on their pages, which means that they provide up to date information on their areas of activities, recent research findings, their publications, and highlights from their scientific career.

The aim is that their contributions extend their magnitude far beyond the Academia boundaries, by attracting the interest of a larger community.

Furthermore, communication means are provided in a form of discussion forums and blogs which enable members, officials, and the general public to discuss certain topics. Some of the topics are open to everyone and some are only open to designated groups.

These many-to-many communications between members could be, for instance, discussions on sensitive matters within a section of the Academia. To illustrate the point, certain members may discuss a candidate which was nominated to become a new member of their section. Thus, these discussions are performed in closed user groups.

Communication means established between members and the general public provide following benefits to the public audience: experts in certain fields can be found, or one wants to prepublish a paper and learn from the comments of other researchers. [2]

\section{AE-Info Implementation}

In [8] the author discusses critical points (author information, administration, access and registration, user practice, design for participation) which should be considered when implementing a wiki system in general. Many of them correspond to our context as well and have been successfully addressed.

AE-Info platform is implemented based on the open source wiki software JSPWiki 2.8. [6]. Enhanced functionality is implemented and provided mostly in form of plug-ins. Member data are stored in a relational database, whereas wiki pages are stored in a file system.

Figure 1 shows a screenshot of AE-Info main page. Anonymous users can navigate, browse, and search the system. However, in order to be able to comment or edit pages, user authentication is required.

An editorial team (consisting of only one member at present) is established to control and enhance content creation process and to support 


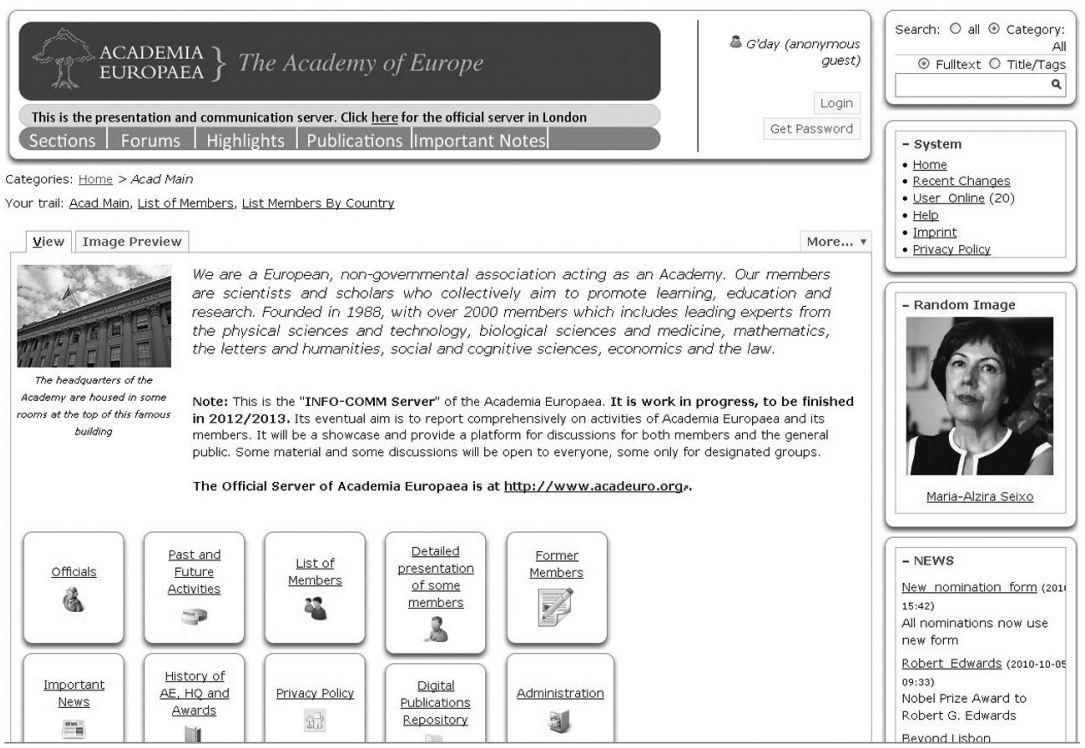

Figure 1. Main page of AE-Info

members when they have problems editing wiki pages.

Main features and approaches to problems outlined in previous sections are discussed in detail in the following sections.

\subsection{AE Section Pages}

AE-Info content is structured mainly based on sections and disciplines of Academia Europeaea. Template based design is applied to generate wiki pages for all 20 sections. Thus, all section pages consist of same structure and lay- out. Template pages were generated automatically through a plug-in that was implemented for this purpose. Our editorial team populated section pages with possessed and gathered data. Thus, an abstraction layer which encapsulates the complexity of creating a section page from scratch (i.e. defining its structure or linking other wiki pages to it) was established. This abstraction layer supported members during the process of content creation. Structuring content based on science disciplines builds up a well suited environment for experts to collaborate with fellows belonging to the same field. Figure 2 visualizes an example of a section page.

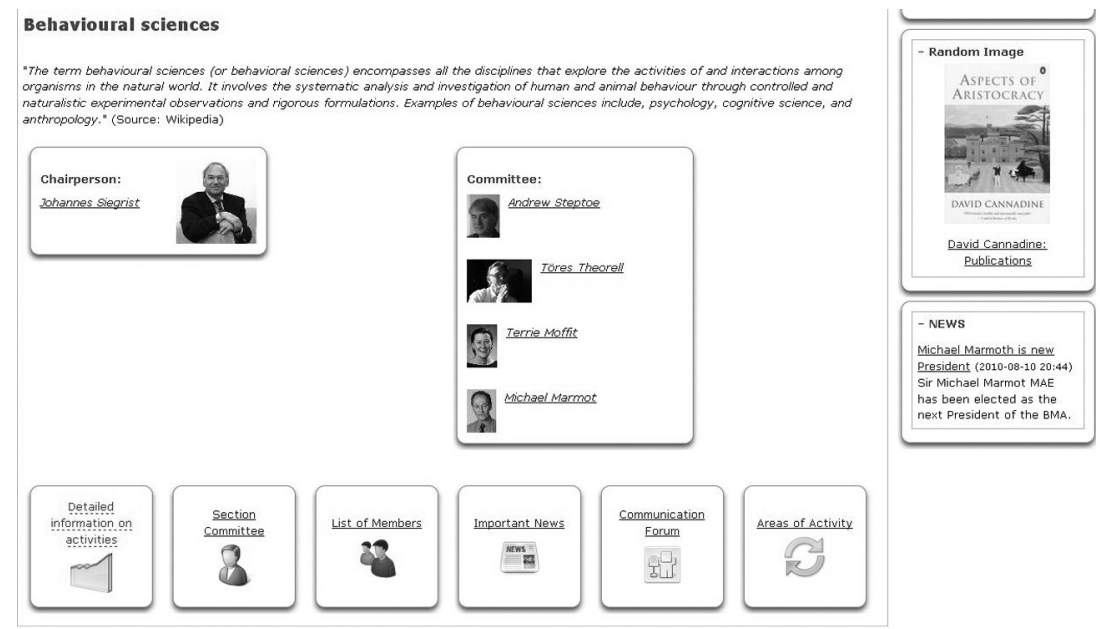

Figure 2. AE-Info section page 
As one can notice from Figure 2, members of a section can collaboratively add/edit content and provide valuable information regarding section committee, activities of a section, or important news. Moreover, members can discuss specific issues with their colleges by clicking on Communication Forum and creating pages for new topics. Worth mentioning is the news plugin (see Figure 2) which presents forthcoming events and activities specific for each section, whereas the same plug-in presents general news in AE-Info main page.

\subsection{AE Member Pages}

Wiki system called AniAniWeb [9] applies the concept of creating home pages using wikis. As the author states it in [9], this concept has been shown to be worth using especially for academics, since their home pages reflect their scientific career and they have to be updated very often. Performing home page updates through a wiki tool is reported to be efficient [9].

The above mentioned facts apply to AE-Info context as well. Thus, each member of Academia Europeaea possesses a home page within AEInfo platform, which can be accessed through a list of all members or within a member section. In order to facilitate the process of home page creation using our wiki system, default member pages were automatically generated. Furthermore, to ease the process of user authentication, default member accounts were generated and sent per email to all members of Academia.

A home page template provides only basic information about a member (essential for contacting members) such as email address, country, section, date of birth, and nationality. However, the predefined structure provides an abstraction layer for members and enables them to add content easily. Help text which describes each step necessary to create/edit wiki pages is also provided. Figure 3 illustrates an example of a member's page with updated member data such as positions held, career achievements, fields of expertise, the member's curriculum vitae.

\subsection{Areas of Activity}

"Areas of activity" is one of the modules presented in each section which comprises all expertise fields belonging to a section. These fields of expertise were automatically extracted from member pages.

As visualized in Figure 4, behind each field of expertise there is a link labeled "Go to". By clicking on this link users can find an expert in that particular field. This special feature is implemented to attract not just members of Academia, but general public as well, which might be interested in detailed information about experts in specific areas.

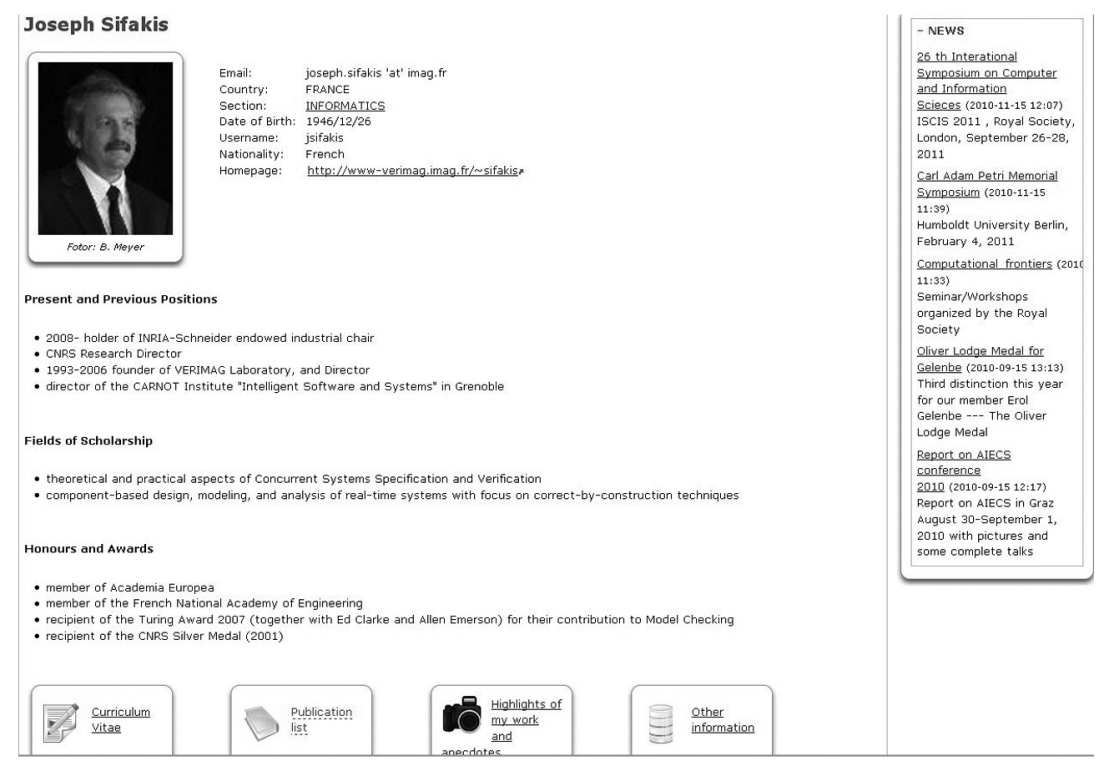

Figure 3. AE-Info member page 


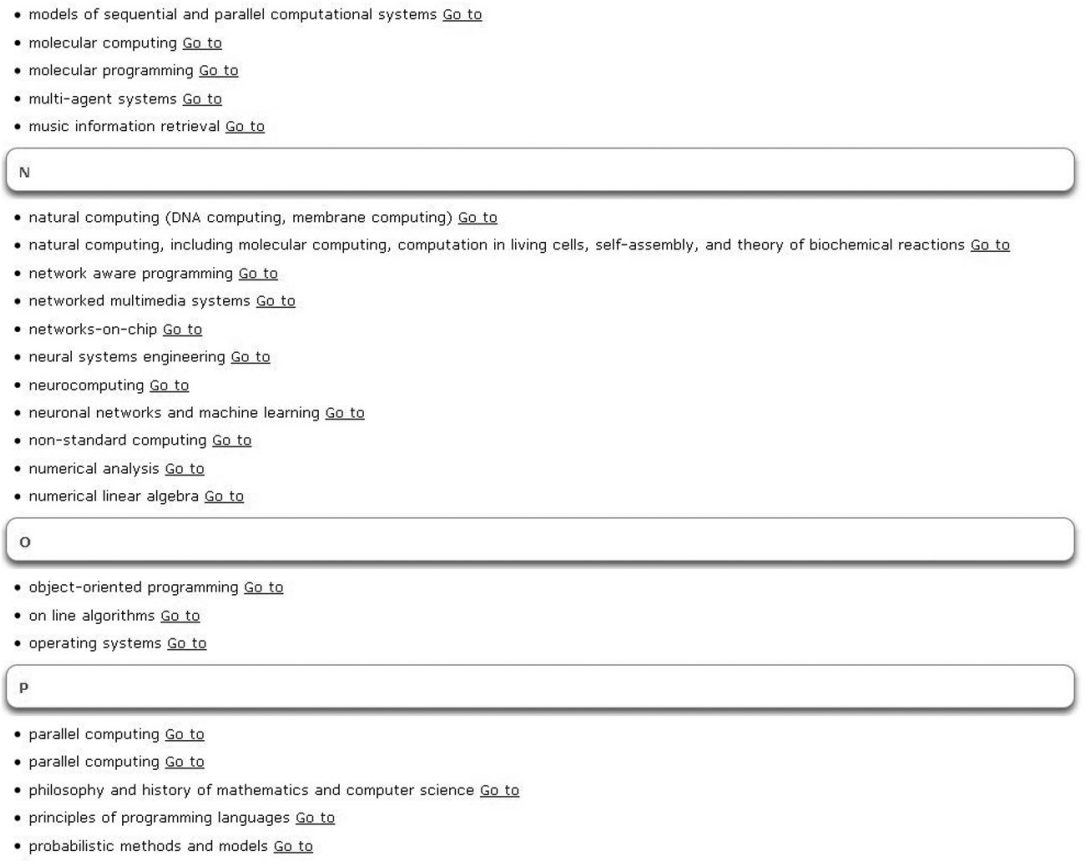

Figure 4. Screenshot of areas of activity of Informatics Section

\subsection{Discussion Forum}

As already mentioned in previous sections, AEinfo platform also offers communication means for members and general public. Wiki pages within AE-Info can be commented by users and discussion topics can be opened as well. However, to add a comment or discuss a topic users have to authenticate.

Introducing terms such as "thread" or "reply" which are used in common discussion forums would challenge our target audience due to their lack of experience with online interactive environments. Therefore, simplified forms of discussion forums are implemented, e.g. so-called blogs.

As it is depicted in Figure 5, user entries are displayed in chronological order. Adding new en- tries corresponds to creating/editing new wiki pages. This simplified concept should ease the process of communication and discussion within AE-Info.

Other communication means are available, but we omit their discussion here.

\subsection{Context Awareness}

Among other features presented in previous sections, AE-Info aims to provide context-aware searching and browsing of content.

In order to facilitate context-aware navigation and browsing within AE-Info, trails and hierarchical breadcrumbs (see Figure 1) are provided on each wiki page. The trail enables users to

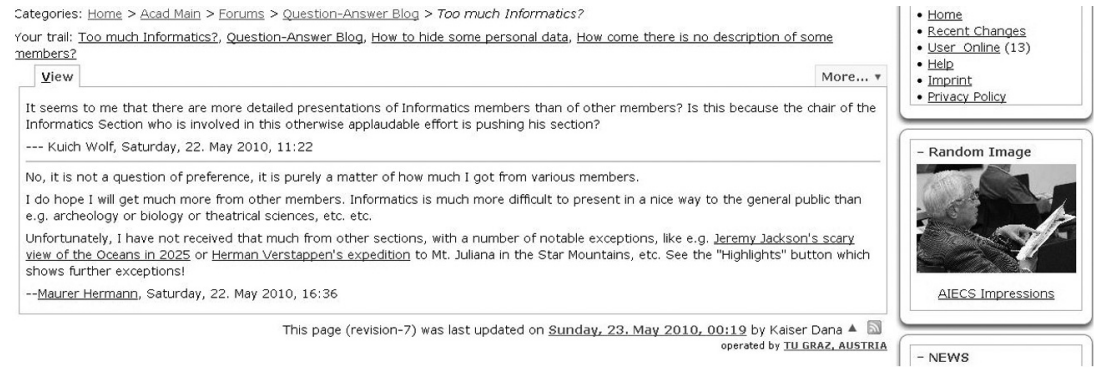

Figure 5. Screenshot of a discussion forum in the form of a blog 
keep track of their last visited pages, whereas breadcrumbs give the position within the hierarchy of wiki pages.

Context-sensitive searching is realized by implementing new mechanisms which complement the existing functionality of the search engine applied in JSPWiki. By providing enhanced search means, users are spared excessive retrieval results; they can perform search in specific areas of the system [13].

A search in AE-Info is performed by using the search field visualized in Figure 1 in the right upper corner. When a user searches for general information, the option "all" should be activated. A more sophisticated option presents the search within categories which can be activated by clicking on "Category". The system is aware of the category a user is currently navigating, so that by activating this option, search results found only in the corresponding category are returned. Furthermore, a full-text search and a tag/title based search are also provided. A metadata search is implemented for retrieving $\mathrm{AE}$ members, so that the retrieved results can be refined by member section or country.

\section{Discussion and Future Work}

This paper presents our experiences, challenges, and respective approaches during the development of a wiki-based system known as Academia Europaea Information and Communication System (AE-Info).

AE-Info aims to present the achievements and activities of Academia Europaea and its members and to provide communication means for members and general public.

AE-Info System was officially launched in May 2010. Our aim is to encourage and motivate our target audience (members of Academia Europaea) to actively participate in our system. To achieve this goal, the following approaches were applied: enhance the content creation process, contact members per email and invite them to update their personal data, complete data in their home pages by our editorial team, and support them during authoring process. As of now, member participation in the process of content creations has not turned out satisfactory. One of the reasons is that our system is still in its early days.

One of our next steps is to study in detail user activities in order to identify user editing behaviors and compare our results with other existing wiki systems.

A new feature that will be offered within AEInfo is an advanced and automated process of nomination of new members. In order to achieve a higher acceptance rate, we are offering two possibilities (online and offline nomination forms) for members to complete the nomination procedure. It is expected that user participation will increase after deploying this new module.

\section{Acknowledgments}

We would like to thank Robert Hoffmann and Dana Kaiser for their valuable inputs and discussions during this work.

\section{References}

[1] T. B. Adler, L. DE Alfaro, I. Pye AND V. RAman, Measuring author contributions to the Wikipedia. Proceedings of the 4th International Symposium on Wikis; 2008 Sep. 8-10; Porto, Portugal.

[2] AE-Info. Academia Europaea Information and Communication System; 2011. www . ae-info.org $[01 / 27 / 2011]$.

[3] R. P. BiuK-AGHAi, L.V.-S. TANG, S. FONG AND Y.W. SI, Wikis as digital ecosystems: An analysis based on authorship. Proceedings of the $3^{\text {rd }} I E E E$ International Conference on Digital Ecosystems and Technologies; 2009 Jun 1-3; Istanbul, Turkey. pp. 581-586.

[4] J. CHAO, Student Project Collaboration Using Wikis. Proceedings of the 20th Conference on Software Engineering Education \& Training (CSEET'07); 2007 Jul. 3-5; Dublin, Ireland.

[5] R. FARZAn, J. DiMicco, D.R. Millen, C. Dugan, W. GEYER AND E. BROWNHOLTZ, Results from deploying a participation incentive mechanism within the enterprise. Proceedings of the Twenty-Sixth Annual SIGCHI Conference on Human Factors in Computing Systems (CHI '08). ACM Press (2008). pp. 563-572.

[6] JSPWiki. JSPWiki - a feature-rich and extensible WikiWiki engine built around the standard J2EE components (Java, servlets, JSP); 2011. http://www.jsp.org [01/27/2011]. 
[7] S.-J. KePP AND H. SchORR, Analyzing Collaborative Learning Activities in Wikis Using Social Network Analysis. Proceedings of CHI 2009, April 4-9, 2009, Boston, Massachusetts, USA.

[8] D.E. O’LEARY, Wikis: From Each According to His Knowledge. Computer 2008. IEEE Computer Society; 41(2): 34-41.

[9] J. RICK, AniAniWeb: a wiki approach to personal home pages. Proceedings of the 2007 International Symposium on Wikis; 2007 Oct. 21-23; Montreal, Canada.

[10] S. SaVAGo AND J. Blat, About the relevance of accessibility barriers in the everyday interactions of older people with the web. Proceedings of the 2009 International Cross-Disciplinary Conference on Web Accessibility (W4A); 2009 April 20-20; Madrid, Spain.

[11] B. Suh, G. Convertino, E.-H. Chi And P. PIROLLI, The Singularity is Not Near: Slowing Growth of Wikipedia. Proceedings of the 5th International Symposium on Wikis and Open Collaboration; 2009 Oct 25-27; Orlando, Florida. pp. 01-10. ACM

[12] L.V.-S. TANG, R.P. BiUK-Aghai And S. Fong, A Method for Measuring Co-authorship Relationships in MediaWiki. Proceedings of the 4th International Symposium on Wikis; 2008 Sep 8-10; Porto, Portugal.

[13] C. Trattner, I. HASANi-MaVRIQI, D. HeliC AND H. LEITNER, The Austrian way of Wiki(pedia)! Development of a Structured Wiki-based Encyclopedia within a Local Austrian Context. Proceedings of the 6th International Symposium on Wikis and Open Collaboration; 2010 Jul 7-9; Gdansk, Poland.

Received: June, 2011 Accepted: November, 2011

Contact addresses:

Ilire Hasani-Mavriqi IICM, Graz University of Technology Inffeldgasse $16 \mathrm{c}$ 8010 Graz, Austria e-mail: ihasani@iicm.edu

Helmut Leitner IICM, Graz University of Technology Inffeldgasse $16 \mathrm{c}$ 8010 Graz, Austria e-mail: hleitner@iicm.edu

Denis Helic KMI, Graz University of Technology Inffeldgasse 21 a 8010 Graz, Austria e-mail: dhelic@tugraz.at

Hermann Maurer IICM, Graz University of Technology Inffeldgasse $16 \mathrm{c}$

8010 Graz, Austria e-mail: hmaurer@iicm.edu
ILIRE HASANI-MAVRIQI is research assistant and $\mathrm{PhD}$ candidate at the Institute for Information Systems and Computer Media (IICM) at Graz University of Technology, Austria. She completed her M.Sc. (with honours) and her B.Sc. in Telematics at Graz University of Technology. Her research interests include web-based information systems, with focus on methods and techniques for improving the navigability within such systems. She is a member of the "Web Application Group" at the IICM. She participated in a number of national and international projects in the fields of information and knowledge management systems and enterprise applications, such as: EPHRAS, APOSDLE, AKL-Kärnten, Net-Files, and AE-Info.

HELMUT LEITNER is senior scientist and manager of the "Web Application Group" at the IICM/TU-Graz (Institute for Information Systems and Computer Media, Graz University of Technology). He is managing and coordinating a highly experienced team of software developers and scientists that has carried out numerous innovative IT projects for large corporations and public institutions in Austria and abroad. Helmut Leitner's professional interests include information and knowledge management systems in general, with particular focus on web applications, intranet solutions and enterprise applications. Before his current position at the IICM, he gained vast experience at the Institute for Hypermedia Systems (HMS), Joanneum Research, Austria, the Austrian Web Application Center (AWAC) of the Austrian Research Centers Seibersdorf, Austria, and the Know-Center Graz, the Competence Center for Knowledge Management in Graz, Austria. He also participated as local project manager in a number of EU projects, such as LIBERATION (an FP4 project on digital libraries), HYPDOC PPP (FP4 project on information systems), APOSDLE (an FP6 IP project), BERNSTEIN (eContentplus project), as well as large projects with the European Space Agency (ESA). Helmut Leitner is also active as lecturer at the TU Graz and has supervised a number of master and $\mathrm{PhD}$ theses.

DENIS HELIC is an associate professor at the Institute for Knowledge Management (KMI) at Graz University of Technology, in Austria. He has a M.Sc. (Dipl. Ing.) in computer technics from the University of Zagreb, Ph.D. in computer science (with honours) from Graz University of Technology, and the post-doctoral lecture thesis for the field "Applied Computer Science" from the Graz University of Technology. His research interests include multimedia and hypermedia information systems, the web, social media and social computation, and information networks. He has published more than 60 research papers in refereed international journals and conferences and won a number of conference best-paper awards. He participated and was the project leader in a number of national and international research projects such as NAVTAG, CORONET, EPHRAS, EuropeMMM, Sprichwort, or Austria-Forum.

HERMANN MAURER has been full professor of computer science at Graz University of Technology in Austria since 1978. In addition, he was director of the Research Institute for Applied Information Processing of the Austrian Computer Society 1983-1998, chairman of the Institute for Information Processing and Computer Supported New Media $1988-2009$ and also director of the Institute for Hypermedia Systems 1990-2006. He was adjunct professor at Denver University 1984-1988, professor of computer science at the University of Auckland, New Zealand, in 1993 (on leave from Graz), back in Graz but remaining adjunct professor in Auckland since the end of 93. His research interests include multimedia and hypermedia information systems, the web, electronic publications and social implications of computers. He has published about 20 books and more than 650 research papers in refereed international journals and conferences. He was project manager of a number of multimillion-dollar undertakings including a patent for optical storage device, the development of a colour-graphic microcomputer (MUPID), an electronic teaching experiment COSTOC, multimedia projects such as "Images of Austria" (Expo'92 and Expo'93) and "Austria-Forum"; various electronic publishing projects such as "PC Library", "Geothek", "J.UCS" and "Brockhaus Multimedial"; responsible for the development of the first and second generation of Web Based Information System Hyperwave. 